Jahrbuch Schweiz - Dritte Welt 1993

\title{
Forschungs-Partnerschaft mit Entwicklungsländern
}

Partenaires dans la recherche avec les pays en développement

Thierry A. Freyvogel

\section{(2) OpenEdition \\ 12 Journals}

Electronic version

URL: http://journals.openedition.org/sjep/1248

DOI: $10.4000 /$ sjep. 1248

ISSN: 1663-9677

Publisher

Institut de hautes études internationales et du développement

\section{Printed version}

Date of publication: 1 février 1993

Number of pages: $215-224$

ISSN: $1660-5926$

\section{Electronic reference}

Thierry A. Freyvogel, « Forschungs-Partnerschaft mit Entwicklungsländern », Schweizerisches Jahrbuch für Entwicklungspolitik [Online], 12 | 1993, Online erschienen am: 30 April 2013, abgerufen am 08

September 2020. URL : http://journals.openedition.org/sjep/1248 ; DOI : https://doi.org/10.4000/sjep. 1248

(c) The Graduate Institute 


\title{
Forschungs-Partnerschaft mit Entwicklungsländern*
}

\author{
Thierry A. Freyvogel
}

\section{Die Ausgangslage}

Ernüchtert, vielleicht sogar enttäuscht, stellte man hiezulande Ende der 80er Jahre fest, dass es trotz mannigfaltiger Anstrengungen bisher nicht gelungen war, in "den Entwicklungsländern" ein eigenständiges Forschungspotential zu schaffen, ein Forschungspotential, das in der Lage gewesen wäre, landeseigene Probleme mit wissenschaftlichen Methoden anzugehen und entsprechende Lösungen vorzuschlagen. In der Tat traf - und trifft - dies wohl für die Mehrzahl der ärmeren Entwicklungsländer zu, insbesondere in Afrika südlich der Sahara. Man folgerte, es müsse mehr getan werden, um diesen Ländern zu ermöglichen, ihre eigenen Forschungskapazitäten aufzubauen (1). In der Schweiz setzten deshalb Ende 1990 die Direktion für Entwicklungszusammenarbeit und humanitäre Hilfe (DEH) des Eidgenössischen Departementes für auswärtige Angelegenheiten (EDA) und die Schweizerische Akademie der Naturwissenschaften (SANW), im Einvernehmen und stellvertretend für die Konferenz der schweizerischen wissenschaftlichen Akademien (CASS), eine Arbeitsgruppe unter der Leitung des Verfassers ${ }^{1}$ ein, die zur Aufgabe hat, ein schweizerisches Konzept zur nachhaltigen Förderung der Forschung in Entwicklungsländern (FFEL) zu erarbeiten.

1 Die Arbeitsgruppe hat ihren Bericht noch nicht fertig gestellt. Für die im vorliegenden Beitrag geäusserten Auffassungen zeichnet allein der Verfasser.

* Cf. résumé p. 263. 


\section{Braucht es Forschung in Entwicklungsländern?}

Forschen, im Sinne des Bestrebens, den Menschen und seine Welt zu verstehen, zählt zu jenen Tätigkeiten des Menschen, die ihn als solchen kenn- und auszeichnen. Es ist nicht einzusehen, weshalb eine derart grundlegende Tätigkeit nur einzelnen Menschengruppen vorbehalten sein, anderen aber vorenthalten werden soltte. Darüber hinaus ist Grundlagenforschung unabdingbarer Ausgangspunkt für zielgerichtete und für angewandte Forschung. Grundlagenforschung stellt somit zugleich kulturelles, humanistisches Schaffen wie auch unerlässliche Voraussetzung für das Angehen praktischer Probleme dar. Selbst Grundlagenforschung ist also kein Luxus.

Zugegeben: Forschung geriet, spätestens seit dem Zweiten Weltkrieg, mehr und mehr, teilweise sehr zu Recht, ins Kreuzfeuer der Kritik. Berechtigt ist die Frage, ob es angebracht sei, Forschung "westlichen" Zuschnitts Entwicklungsländern gewissermassen aufzudrängen, beziehungsweise sie auch dort anzusiedeln. Die Antwort kann nur bejahend lauten; ohne Anwendung wissenschaftlicher Methoden lassen sich die Probleme, welche die Menschheit zunehmend und weltweit bedrohen, nicht mehr, und sicher nicht rechtzeitig lösen. - Nicht zu beantworten ist hier die andere Frage, inwieweit und in welcher Weise Forschungsergebnisse überhaupt anzuwenden seien. Offen bleibt schliesslich auch, ob nichtwestliche Menschen mit den Ergebnissen der Forschung verständiger werden umzugehen wissen, als dies bisher den Menschen der Industriestaaten gegeben war.

Damit ist gesagt, dass es auch in den Entwicklungsländern eigenständige Forschung braucht, und dass dazu Grundlagen-, zielgerichtete und angewandte Forschung zählen. Es liegt im Interesse auch der Industrieländer, dass die efforderlichen Kapazitäten errichtet werden. Eine allein auf Industrienationen beschränkte wissenschaftliche Zusammenarbeit genügt nicht (mehr).

\section{Zu den heutigen Forschungskapazitäten in Entwicklungsländern}

Anerkanntermassen ist es um das Forschungspotential vieler Entwicklungsländer, namentlich in Afrika südlich der Sahara, schlecht bestellt (2). Die Bedeutung, welche der wissenschaftlichen Forschung am Ausgang des 20. Jahrhunderts zukommt, ist nicht erkannt; sie wird von den Entscheidungsträgern nicht gesehen. Hinzu kommt häufig politische Unstabilität. Aufgrund beider Umstände fehlt es weitgehend an nationaler Forschungspolitik.

Dem entsprechen die unsicheren Berufsaussichten für Forscher und wissenschaftliches Personal. Die Löhne reichen für das Leben kaum aus; Nebenverdienste sind unumgänglich. In ihrer Eigenschaft als Forscher haben die Wissenschafter wenig Kontakt zur eigenen Bevölkerung; sie nehmen als solche an deren Sorgen keinen Anteil (3). Entsprechend gering ist das Ansehen, das sie in der Öffentlichkeit geniessen; entsprechend gering ist auch ihre Motivation, For- 
schungsarbeit zu leisten, die der Entwicklung des eigenen Landes etwas bringen könnte.

Häufig fehlt es überdies an der erforderlichen Ausstattung, gleichgültig ob es sich um Literatur oder Ausrüstungsgegenstände handle. Wichtiger noch als solche Mängel dürfte die wissenschaftliche Isolation sein, die Unmöglichkeit, mit kompetenten Kollegen des eigenen oder anderer Fächer regelmässigen Umgang zu pflegen. Gerade bei Wissenschaftern, die ihre Ausbildung zum Forscher im einen oder andern Industrieland erfuhren, wirkt sich diese Vereinsamung oft verheerend aus.

\section{Zu den erforderlichen Kapazitäten in Industrieländern}

Das berüchtigte "Publish or perish" gilt in der Schweiz nicht weniger als in andern Industrieländern. In aller Regel sieht sich der Forscher bei uns gedrängt, möglichst rasch möglichst viel zu veröffentlichen, und zwar in "international anerkannten Zeitschriften", und dies so, dass die eigenen Titel möglichst häufig zitiert werden. Modern muss man sein. Zeitraubende Feldarbeit ist unergiebig, marktwirtschaftlich Irrelevantes tunlichst zu meiden. Der Gedanke daran, dass die erzielten Forschungsresultate auch in Entwicklungsländern Verwendung finden könnten, ist wenig verbreitet. - Möglicherweise überzeichnen diese kritischen Äusserungen die Realität teilweise. Dessen ungeachtet helfen sie vielleicht, einige Schwachstellen des Wissenschaftsbetriebs, wie wir ihn von Industrieländern kennen, hervortreten zu lassen. Schwachstellen, die unsere Forscher daran hindern, ihre Arbeit unter anderem auch im Dienst der weltweiten, langfristigen Entwicklung zu sehen.

Interdisziplinarität wird zwar häufig gefordert, jedoch wenig praktiziert und nur ausnahmsweise schon gelehrt. Wie zahlreich sind etwa Biologen, die von $\mathrm{Ge}$ schichte, Wirtschafts- oder Sozialwissenschaften genug verstehen, um mit den entsprechenden Fachvertretern wirkungsvoll zusammenspannen zu können ? Und doch ist die Lösung dringender weltweiter Probleme mittel- bis langfristig nur in Fakultäten-übergreifender Weise möglich!

Selbst innerhalb des eigenen Faches werden die unterschiedlichen Disziplinen häufig unausgewogen dargeboten. Der stets lauter werdende Ruf nach Erhaltung der Umwelt, Schutz der Natur und damit auch der Biodiversität (4) hat zur Einsicht geführt, dass es heute weltweit an Taxonomen mangelt. Die nun über Jahre dauernde starke Förderung der Molekularbiologie und der damit zusammenhängenden neueren Richtungen erfolgte zu Lasten älterer, deswegen jedoch nicht unnötiger Disziplinen. - Häufig fehlt es Studien-Abgängern an praktischer Erfahrung. An die Fähigkeit unserer Wissenschafter, Entscheidungsträger zu überzeugen oder sich einer breiteren Öffentlichkeit verständlich mitzuteilen, sei bloss im Sinne eines lange schon bekannten Wunschtraums erinnert.

Man täusche sich nicht: eine nachhaltige Förderung der Forschung in Entwicklungsländern erheischt tiefgreifende Veränderungen im universitären Forschungs- und Lehrbetrieb auch auf Seiten der Industrieländer. Dass dies solchen zum Vorteil gereichen könnte, scheint auf der Hand zu liegen; 
nachhaltige Förderung der Forschung in Entwicklungsländern dürfte langfristig gesehen zu einer willkommenen und notwendigen Stärkung auch des Forschungsplatzes Schweiz führen.

\section{Was hat die Schweiz bisher zur Förderung von Forschung in Entwicklungsländern beigetragen?}

Für die Direktion für Entwicklungszusammenarbeit und humanitäre Hilfe (DEH) ist das Bundesgesetz über die internationale Entwicklungszusammenarbeit aus dem Jahre 1976 massgebend. Dieses strebt "besser ausgewogene Verhältnisse in der Völkergemeinschaft" an. Zentrales Anliegen der DEH ist die bestmögliche Entfaltung des Menschen in seinem natürlichen, kulturellen und gesellschaftlichen Umfeld. Die DEH unterstützt die ärmere Bevölkerung, damit diese ihre Grundbedürfnisse für Nahrung, Wohnung, Gesundheit und Erziehung befriedigen kann (5). Für die DEH steht demzufolge nicht die Forschung sondern die Entwicklung im Vordergrund. Erst, wo Entwicklung Forschung voraussetzt, kann die DEH sich für die Forschung in Entwicklungsländern einsetzen. Erst in jüngster Zeit, als die DEH die Forschung als bedeutendes Mittel zur Entwicklung erkannte, begann sie, sich für die Förderung der Forschung in Entwicklungsländern stark zu machen.

Gleichwohl hat die DEH, wie eine 1991 abgeschlossene interne Studie ergab, wesentlich mehr für Forschung in Entwicklungsländern aufgewendet, als allgemein angenommen werden konnte. Allein im Jahre 1991 wurden ca. 80 Millionen Franken für die Finanzierung von Projekten mit einer Forschungskomponente ausgegeben. Fast die Hälfte entfiel auf die Landwirtschaft, vorab in Ostafrika und in asiatischen Regionen. In der genannten Zahl nicht berücksichtigt sind die für Stipendien, Aus- und Weiterbildung eingesetzten Gelder. Trotz dieser Konzentration auf bestimmte Fachdienste und auf ausgesuchte geographische Regionen spricht die genannte Studie bezüglich der Förderung von Forschung und Wissenschaft von einer "heterogenen Situation", ohne "klare strategische Ausrichtung". Bemerkenswert ist ferner, dass nur in einem runden Viertel der Fälle schweizerische Forschungsinstitutionen an DEH-Projekten mit einer Forschungskomponente beteiligt waren. - Die DEH ist gegenwärtig damit beschäftigt, ihre Strukturen zu überholen und die internen Richtlinien zu überarbeiten; es darf erwartet werden, dass es ihr gelingt, die eigenen Aktivitäten noch wirkungsvoller als bisher in den gesamtschweizerischen Rahmen einzugliedern.

Die Eidgenössische Stipendienkommission für ausländische Studierende (ESKAS) vergibt, in Übereinstimmung mit dem entsprechenden Bundesgesetz aus dem Jahre 1987, von den jährlich rund hundert neuen Stipendien deren 50 an Leute aus Entwicklungsländern. Da diese Stipendien, im Gegensatz zu jenen an Studierende aus Industrieländern, über mehrere Jahre verlängert werden können, liegt die Gesamtzahl von Studierenden aus Entwicklungsländern an schweizerischen Hochschulen jederzeit um die 300. Im Ganzen werden die Studien mit Erfolg absolviert, und die Studierenden kehren mit einem schwei- 
zerischen Diplom, Lizentiat oder Doktorat in ihre Heimat zurück. - An dieser Stelle darf erwähnt werden, dass einzelne Kantone, wie beispielsweise Basel-Stadt, mit ähnlichem Erfolg vergleichbare Aktionen durchführen.

Die Probleme beginnen mit der Wiedereingliederung im Heimatland. Obschon die Stipendien auf den ausdrücklichen Wunsch der betreffenden Entwicklungsländer erteilt werden, sind die ursprünglich vorgesehenen Posten bei der Rückkehr der Stipendiatin oder des Stipendiaten möglicherweise entweder nicht verfügbar oder so schlecht dotiert, dass die Betroffenen es vorziehen, einer andern Beschäftigung nachzugehen oder ihr Land wieder zu verlassen. Diese Gefahr könnte verringert werden, wäre die ESKAS befugt, unter bestimmten Bedingungen ehemalige Stipendiaten in angemessener Weise weiter zu fördern. Ebenfalls denkbar ist die Eingliederung Ehemaliger in internationale Forschergruppen, wie sie weiter unten noch zu beschreiben sind.

Die beiden Eidgenössischen Hochschulen und alle kantonalen Universitäten unterhalten zahlreiche Kontakte mit sehr diversen Stellen in nahezu allen Entwicklungsländern. Einen ersten, einzigartigen Überblick gibt eine von der Arbeitsgruppe FFEL in Auftrag gegebene und 1992 fertiggestellte Studie (6), die über 250 schweizerische Institutionen und über 700 Schweizer Forscherinnen und Forscher auflistet, die an rund 370 Forschungs-Projekten beteiligt sind. Am häufigsten sind die Verbindungen zu West- und Ostafrika; doch sind auch jene nach Ostasien und nach Lateinamerika nicht unbedeutend. Natur- und Sozialwissenschaften sind besonders stark vertreten; Medizin, Wirtschaftswissenschaften, sowie Ökologie und Umwelt finden sich ebenfalls verhältnismässig oft berūcksichtigt.

Dass in zahlreichen Fällen der Schweizerische Nationalfonds an der Finanzierung der Projekte beteiligt ist, sei hier lediglich in Erinnerung gerufen.

Indessen tragen diese reichhaltigen Tätigkeiten mehr zur Veröffentlichung wissenschaftlicher Ergebnisse europäischer Kollegen bei als zur Festigung des ortsansässigen Forschungspotentials. Hand in Hand damit geht die Tatsache, dass, mit einer Ausnahme, keine schweizerische Hochschule in der Lage ist, jene ihrer Dozenten und Mitarbeiter zu nennen, die Forschung in Verbindung mit Entwicklungsländern betreiben, und dass die schweizerischen Forscher, wenn überhaupt, in dieser Hinsicht voneinander sehr wenig wissen.

Die Schweizerische Akademie der Naturwissenschaften (SANW) betreibt in Adiopodoumé, nahe Abidjan, der Hauptstadt der Côte d'Ivoire, seit 1951 das Centre Suisse de Recherches Scientifiques (CSRS). Ursprünglicher Zweck des Unternehmens war, jungen Schweizer Wissenschaftern die Möglichkeit zu verschaffen, ihrer Forschung unter tropischen Verhältnissen nachzugehen. Im Vordergrund standen lange Jahre die Botanik, Zoologie, besonders Parasitologie, Ethnologie und Bodenkunde. Seit wenigen Jahren tritt nun die Forschungszusammenarbeit mit der Côte d'Ivoire in stets stärkerem Masse in den Vordergrund. Gezielt wird, im Einvernehmen mit den ivorianischen Behörden, versucht, jüngere ivorianische Forscher zur Mitarbeit zu gewinnen. Gleichzeitig wurde auch das Forschungsprogramm gestraftt; es gilt nun vorwiegend dem "Naturraum", der "Ernährung" und der "Parasitologie" (7). 
Einen Schritt weiter ist das Schweizerische Tropeninstitut (STI), Basel, gegangen, das sein 1957 eröffnetes, ehemaliges Feldlaboratorium über die Jahre hindurch konsequent in den Verbund der Laboratorien des National Institute for Medical Research (NIMR) Tansanias überführte und nun, ebenfalls im Einvernehmen und unter aktiver Mitbeteiligung der Landes-Behörden, als das Ifakara Centre des NIMR weiterführt. In Entsprechung mit dem Aufgabenbereich des STI werden am Ifakara Centre gegenwärtig vor allem Medizinische Parasitologie und "Health Systems Research" betrieben. Bemerkenswert ist dreierlei: die Hilfeleistung des Centre beim Betrieb des Gesundheitswesens im Distrikt, womit der Kontakt zur ortsansässigen Bevölkerung gegeben ist; die wachsende Zahl afrikanischer Kollegen und Mitarbeiter in verantwortungsvoller Position; und die internationale Forschungs-Zusammenarbeit mit Organisationen der Vereinten Nationen und unter Beteiligung von Forschern aus andern europäischen Industrieländern (8).

CSRS und Ifakara Centre bieten, vorab wegen ihrer Langjährigkeit, höchst interessante Möglichkeiten bezüglich der Forschungsförderung in ihren Gastländern und möglicherweise darüber hinaus in deren Nachbarländern. Sie bleiben aber beide bis auf weiteres auf ihre Träger-Institutionen, in den vorliegenden Fällen die SANW bzw. das STI, angewiesen. Es versteht sich von selbst, dass diese Institutionen funktionstüchtig erhalten werden müssen, wenn sie ihre unverzichtbaren Aufgaben gegenüber den genannten Aussenstationen erfüllen sollen. Ähnliches gilt sinngemäss für andere bewährte Zentren in der Schweiz wie beispielsweise das Institut universitaire des Hautes Etudes Internationales (IUHEI), das Institut des Etudes du Développement (IUED), beide in Genf, das Geographische Institut der Universität Bern (GIUB), das Zentrum für Internationale Landwirtschafts-Forschung (ZILF), sowie die Nachdiplom-Kurse für Probleme der Entwicklungsländer (NADEL), die beiden letzten an der Eidgenössischen Technischen Hochschule Zürich (ETHZ). Der Aufwand, der allein zur Sicherung der Betriebsmittel mehrerer der genannten Institutionen geleistet werden muss, steht vielfach in keinem Verhältnis zum Erfolg. Hier wird in der Schweiz am falschen Ort "gespart", nicht aus Böswilligkeit, sondern viel eher, weil die zahlreichen zuständigen Stellen auf Kantons- und Bundesebene zu Problemlösungsorientierter unkomplizierter Zusammenarbeit (noch) nicht fähig sind.

Nicht zu vernachlässigen sind weitere Nicht-Regierungs-Organisationen ("Non-governmental organisations" NGOs), welche in Verbindung mit Entwicklungsprojekten unterschiedlichster Art Angewandte Forschung betreiben oder unterstützen. Vor allem aber darf die Forschung in Entwicklungsländern der PrivatIndustrie nicht vergessen werden, die häufig auch mit Fortbildung nationaler Forschungskräfte verknüpft ist, die jedoch im Rahmen des vorliegenden Beitrags nicht behandelt werden kann.

\section{Schlussfolgerungen}

Fassen wir zusammen: Auch Entwicklungsländer benötigen eigenständige Forschung. Ausserdem stehen dringende Probleme weltweit an, deren Lösung nur 
unter Anwendung wissenschaftlicher Forschung in internationaler Zusammenarbeit möglich scheint. Es liegt demnach im Interesse auch der Industrieländer, mitunter der Schweiz, beim Aufbau der benötigten Forschungskapazitäten in Entwicklungsländern mitzuwirken. Bisher wurde zwar in dieser Richtung einiges unternommen, das jedoch bezüglich des Aufbaus des Forschungspotentials in Entwicklungsländern kaum zum Ziel führte. Einerseits sind die Voraussetzungen für den Aufbau nationaler Forschungspotentiale in Entwicklungsländern weder bei innen noch in den Industrieländern vollumfänglich gegeben; anderseits wurde dieser Aufbau des erwünschten Potentials in Entwicklungsländern noch kaum je explizit zum Ziel der Forschungs- und/oder allgemeinen Entwicklungszusammenarbeit gemacht.

Demnach braucht es eine Strategie, oder zum mindesten ein Konzept, expressis verbis "zur Förderung der Forschung in Entwicklungsländern". In Anbetracht dessen, was bis jetzt geleistet worden ist, und der Erfahrungen, die dabei gewonnen wurden,

- ist fortzuführen und zu verbessern, was sich bisher als geeignet erwies;

- ist die schweizerische wissenschaftliche Gemeinschaft für den Forschungsbedarf in Entwicklungsländern zu sensibilisieren;

- sind die zuständigen Stellen der eidgenössischen Verwaltung zur zielgerichteten, departementsübergreifenden Zusammenarbeit einzuladen;

- ist in weitesten Kreisen zu informieren;

- ist mit Blick auf das Hauptziel zu koordinieren;

- sind die Mittel zu konzentrieren;

- sind die vorgesehenen schweizerischen Leistungen im Verbund mit denjenigen anderer Industrienationen zu erbringen;

- ist die Wirkung der Tätigkeiten mit Blick auf das Hauptziel laufend zu überprüfen und die Strategie gegebenenfalls anzupassen; und

- ist überdies ein neuer Ansatz zu suchen.

\section{Das "Spezialprogramm"}

Grundgedanke des neuen Ansatzes ist, nicht zu helfen, sondern Probleme gemeinsam zu lösen. Es gibt drängende Fragen, die Entwicklungs- und Industrieländer gleichermassen angehen und belasten, beispielsweise die weltweite demographische Entwicklung, die Ernährung, Gesundheit, Schulung, die Umwelt oder die Energie.

Zumindest liessen sich mit Methoden wissenschaftlicher Forschung Teilprobleme anpacken und von partnerschaftlich zusammengestellten Gruppen bearbeiten. "Partnerschaftlich" bedeutet im vorliegenden Zusammenhang zweierlei. Zum ersten paritätisch: gleich viele Wissenschafter aus den teilnehmenden Entwicklungs- und Industrieländern; zum zweiten alle Funktionen umfassend: Forschungsgruppen der erforderlichen Grössenordnung umfassen neben Akademikern auch Techniker, Verwaltungsleute und Hilfspersonal. 
Problemkreise dieses Ausmasses lassen sich nicht innert weniger Jahre bewăltigen. Was immer dafür unternommen werden soll, muss langfristig, über Jahrzehnte, wenn nicht über Generationen angelegt sein. - Keines der als Beispiele erwähnten Probleme ist Angelegenheit einer einzelnen Disziplin; alle sind sie äusserst vielschichtig. Die Unterfangen müssen demnach von Anbeginn an interdisziplinär geplant und durchgeführt werden. In allen genannten Fällen spielen neben den unmittelbar betroffenen naturwissenschaftlichen Fächern Wirtschaftswissenschaften und Soziologie eine entscheidende Rolle; dass damit Medizin, Ethnologie, Theologie, Geschichte oder noch manche andere Fächer, die gewohnheitsmässig als geisteswissenschaftliche bezeichnet werden, nicht ausgeschlossen sind, muss an dieser Stelle nicht weiter ausgeführt werden. - Die fraglichen Probleme betreffen nicht einzelne Nationen allein; sie müssen deshalb im übernationalen Kontext, regional, angegangen werden.

Was an Einzelprojekten unternommen werden soll, hat bestimmten Kriterien zu genügen. Jedes Projekt muss einem Bedürfnis aller Partner entspechen, also sowohl demjenigen in der interessierten Entwicklungsländer-Region wie auch der beteiligten Industrieländer. Jedes Projekt muss die vorhandenen Forschungskapazitäten berücksichtigen und dem Erfordernis der kritischen Masse genügen. Es muss für Forscher aus beiden Lagern attraktiv sein, also neben angewandter Forschung von unmittelbarer Entwicklungs-Relevanz auch Grundlagenforschung beinhalten. Es soll das Lebens-Niveau der ortsansässigen Bevölkerung anheben helfen. Es muss umweltverträglich sein. Und selbstverständlich muss es, nach Ablauf einer gewissen Zeit, eine positive Auswirkung auf die zu schaffenden Forschungspotentiale nachweisen können.

Die eingangs erwähnte Arbeitsgruppe wird als zentrales Thema des Spezialprogramms den "Umgang mit natürlichen Ressourcen" vorschlagen. Das Thema ist von hoher Aktualität; es dürtte davon auch in den kommenden Jahrzehnten nichts verlieren. Es umfasst Bereiche wie Sonnenenergie, das Erhalten der Böden, Wasser und Gewässerschutz, Luftverschmutzung; Landschaftspflege einschliesslich Erholung und Tourismus, die Pflanzenwelt einschliesslich der Wälder, die Tierwelt und die Biodiversität. Es umfasst auch das "human resources development", also den Umgang mit und die Förderung von menschlichen Fertigund Fähigkeiten. - Dieses gewählte allgemeine Thema ist wohl noch zu weit gefasst. Weitere Eingrenzungen sollen jedoch erst im Gespräch mit den künftigen Partnern vorgenommen werden.

Neben der fachlichen brächte möglicherweise noch eine andere Einschränkung, nämlich eine Konzentration der Tätigkeiten auf eine oder auf wenige geographische Regionen, Vorteile mit sich. Wenn die in Aussicht zu nehmenden Aktivitäten nicht allzu weit zerstreut erfolgten, liesse sich ihre Wirkung auf den Aufbau der nationalen Forschungspotentiale wohl eher und rascher erfassen. Für bestimmte einzelne Regionen lassen sich gewichtige Argumente anführen, wie etwa die Dichte der bereits bestehenden Kontakte zur Schweiz, die in der Schweiz vorhandenen besonderen Kapazitäten, das Bestehen guter Ausbildungszentren in den regionalen Entwicklungsländern, das Bestehen eines schweizerischen Zentrums, bereits erworbene, positive Erfahrung mit internationaler Forschungs- 
Zusammenarbeit und manche andere mehr. Anderseits ist nicht zu leugnen, dass sich je nach der Wahl der geographischen Konzentrationsgebiete erfahrene Schweizer Forschungsgruppen ausgeschlossen sehen könnten. - Auch in diesem Punkt werden möglicherweise Gespräche mit potentiellen Partnern eine zwar pragmatische, aber brauchbare Lösung bringen.

Nicht die alles entscheidende, doch die alle bewegende Frage ist diejenige nach der Finanzierung. Anzustreben ist eine gemischte Finanzierung des hier umrissenen Spezialprogramms. Nach dieser Vorstellung kämen im wesentlichen die DEH für die Mitarbeiter aus den Entwicklungsländern und die schweizerischen Hochschulen und kantonalen Universitäten (u.U. mit Unterstützung des Nationalfonds) für ihr eigenes Personal auf. Je nach den anfallenden Forschungsaufgaben dürfte ausserdem mit der Unterstützung durch die jeweils zuständigen Bundesämter gerechnet werden. In einer Anfangsphase wären deshalb keine erheblichen zusätzlichen Mittel erforderlich; heute bereits fliessende Quellen hätten lediglich einen Teil ihrer Mittel neu zu disponieren. Wohl aber müsste der Start gewährleistet sein; hiefür sollen demnächst die entsprechenden Kontakte hergestellt werden.

Teilweise von der Art der Finanzierung werden die Zusammensetzung und die Angliederung des Führungsgremiums abhängen. Was benötigt wird, ist eine ziemlich unabhängige, mit verhältnismässig weitgehenden Kompetenzen ausgestattete Gruppe erfahrener Leute, welche das Unternehmen allen Interessierten bekanntgeben, die eingehenden Projekt-Vorschläge beurteilen, die Mittel zusprechen, den Ablauf der Tätigkeiten verfolgen, die Ergebnisse auswerten, und die für die gesamte Strategie massgebenden Kreise beraten.

\section{Ausblick}

Die hier umrissene Strategie und das ansatzweise skizzierte Spezialprogramm ergeben sich, wie es scheint, folgerichtig aus den bisherigen Erfahrungen. Gleichwohl sind sie anspruchsvoll. Die von der Arbeitsgruppe so weit entwickelten Vorstellungen erhielten bisher seitens der Behörden wie seitens der schweizerischen und der ausländischen wissenschaftlichen Gemeinschaft unerwartet grosse Zustimmung. Doch bleibt noch manche Frage zu klären und muss mit zu erwartenden wie unerwarteten Schwierigkeiten, in den Partnerländern wie in der Schweiz, mit Sicherheit gerechnet werden. Doch wo ein Wille ist, ist auch ein Weg. Ob die Schweiz ihn einschlagen und konsequent gehen will, wird die Zukunft weisen.

\section{Quellennachweis}

1. Dritte Welt und Naturwissenschaften / hrsg. von d. Freiburg. Naturforschenden Ges. Freiburg (Schweiz), Univ. Verlag, 1990

2. Manifest August 1992 / hrsg. von der Arbeitsgruppe FFEL. Erhältlich bei: SANW, Generalsekretariat, Bärenplatz 2, 3011 Bern 
3. KABOU Axelle, Et si l'Afrique refusait le développement ?, Paris, L'Harmattan, 1991

4. Education and Science for Maintaining Biodiversity / hrsg. von der Schweizerischen Nationalen UNESCO-Kommission - Bern, 1992

5. Leitbild der DEH (Schriften der DEH 1/1991). Zu beziehen bei: Informationsdienst DEH, 3003 Bern

6. Scientific Cooperation with Developing Countries: Who does what in Switzerland ? (A preliminary survey of institutions, research projects, researchers) / hrsg. von IUED, SANW, DEH. Erhältlich bei: Institut universitaire d'études du développement IUED, Centre de documentation, Case postale 136, 1211 Genève 21

7. 40 Jahre CSRS, in: Bulletin SAGW/SANW XVII/1992/1, p.68. Erhältlich bei: SANW, Generalsekretariat, Bärenplatz 2, 3011 Bern

8. Ifakara Centre, Annual Report 1991-92. Erhältlich bei: Schweizerisches Tropeninstitut, Bereich GWE, Postfach, 4002 Basel 